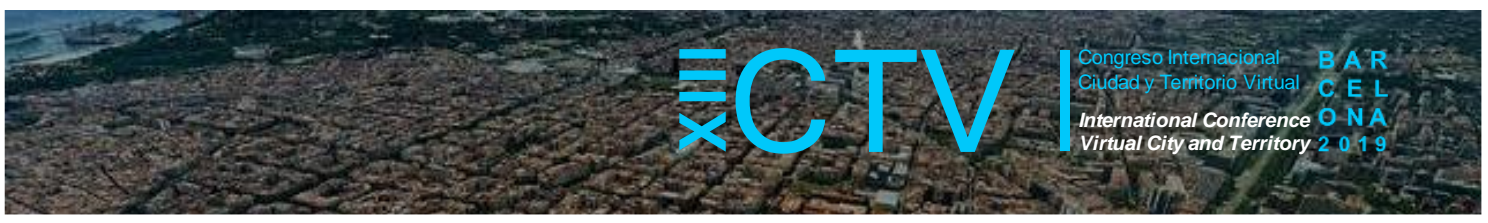

\title{
LA RECUPERACIÓN DE PLUSVALÍAS URBANAS Y LA MATERIALIZACIÓN DE JUSTICIA SOCIOESPACIAL EN BOGOTÁ (COLOMBIA) Y SAO PAULO (BRASIL)
}

\author{
Pérez-Moreno, Oscar ${ }^{1}$
}

Remisión inicial: 2019-06-16; Remisión definitiva: 2019-10-20; Publicación: 2019-12-21

Citación: Pérez-Moreno, O. (2019). La recuperación de plusvalías urbanas y la materialización de justicia socioespacial en Bogotá (Colombia) y Sao Paulo (Brasil). En XIII CTV 2019 Proceedings: XIII International Conference on Virtual City and Territory: "Challenges and paradigms of the contemporary city": UPC, Barcelona, October 2-4, 2019. Barcelona: CPSV, 2019, p. 8640. E-ISSN 2604-6512. DOI http://dx.doi.org/10.5821/ctv.8640

\begin{abstract}
Resumen
La ponencia analiza la relación entre la recuperación de plusvalías urbanas y la materialización de justicia socioespacial en las ciudades de Bogotá (Colombia) y de Sao Paulo (Brasil), estudiando dos casos relevantes de recuperación de plusvalías urbanas en dichas ciudades: la Distribución Equitativa de Cargas y Beneficios (DECB) definida en el Decreto Distrital 562 de 2014 en Bogotá y la Operación Urbana Consorciada Água Espraiada (OUCAE) aprobada mediante la Ley Municipal 13.260 de 2001 en Sao Paulo. La materialización de justicia socioespacial es evaluada a partir de los avances en la disminución de cinco problemáticas relacionadas a los rasgos particulares de los procesos de urbanización en América Latina: 1. La disminución de la apropiación privada de la valorización de la tierra resultante de la actuación del Estado; 2. La consolidación de los conceptos de otorga onerosa del derecho de construir y de distribución equitativa de cargas y beneficios; 3. La alteración de los patrones de distribución de la infraestructura urbana; 4. La reducción de los efectos negativos de la segregación socioespacial y 5 . La disminución de la informalidad en el acceso al suelo urbano. La pregunta central que orienta la ponencia busca responder si en la DECB en Bogotá y en la OUCAE en Sao Paulo se presentaron avances en la materialización de formas de justicia socioespacial asociadas a la disminución de las problemáticas citadas. La hipótesis utilizada para responder a la pregunta central consideró que, en la DECB implementada en Bogotá, y en la OUCAE desarrollada en Sao Paulo, se concretaron avances en la materialización de formas de justicia socioespacial, debido a que, en su implementación, la recuperación de plusvalías urbanas fue considerada como un proceso que involucró acciones anteriores y posteriores a la recuperación, prevaleciendo el principio distributivo y la meta redistributiva. La metodología tuvo un énfasis comparativo y tres niveles de análisis: En el nivel general, se examinaron los conceptos, leyes e instrumentos relacionados a la temática de la investigación en Colombia y Brasil. En el nivel específico, se analizó el impacto de los instrumentos de recuperación de plusvalías urbanas estudiados en la materialización de justicia socioespacial en Bogotá y Sao Paulo, por medio de tres momentos: la acción anterior a la recuperación; la acción de recuperación y la acción posterior a la recuperación. En el nivel síntesis, se hizo una comparación de los resultados de la materialización de la justicia socioespacial. La hipótesis propuesta en la ponencia fue confirmada parcialmente, considerando que, en los casos estudiados, la acción anterior a la recuperación de plusvalías urbanas estuvo caracterizada por la distribución desigual de la infraestructura urbana y por la valorización diferenciada de la tierra. En esta acción se encontró también la necesidad fundamental de definir mecanismos que busquen la disminución de las desigualdades en la distribución de la renta y la riqueza en la sociedad, teniendo en cuenta los rasgos característicos de este primer momento. En el caso de la acción de recuperación de plusvalías urbanas, se presentaron avances en el combate a la apropiación privada de la valorización de la tierra y en la consolidación del principio distributivo. Finalmente, se encontró que un aporte práctico resultado del análisis de la acción posterior a la recuperación de plusvalías urbanas, está relacionado a que los recursos obtenidos deben ser prioritariamente destinados para atender a la población de menores ingresos, ya sea en las mismas áreas donde fueron realizados los proyectos, como en las otras áreas de la ciudad que no son beneficiadas por los presupuestos generales de los municipios.
\end{abstract}

\section{Abstract}

The present paper brings an analysis between land value capture and the materialization of socio-spatial justice in Bogotá (Colombia) and in São Paulo (Brazil), by the exploration of two relevant cases: Equitable Distribution of Charges and Benefits (Distribución Equitativa de Cargas y Beneficios - DECB -in Spanish) in Bogotá, and the Consortiated Urban Operation Água Espraiada (Operação Urbana Consorciada Água Espraiada - OUCAE -, in portuguese) in São

\footnotetext{
${ }^{1}$ Arq., Mag. en Estudios Urbano-Regionales y Dr. en Arquitectura y Urbanismo. Docente de cátedra Universidad de
} Antioquia (Colombia), https://orcid.org/0000-0002-9866-9444. * Correo de contacto: oscare.perez@udea.edu.co 


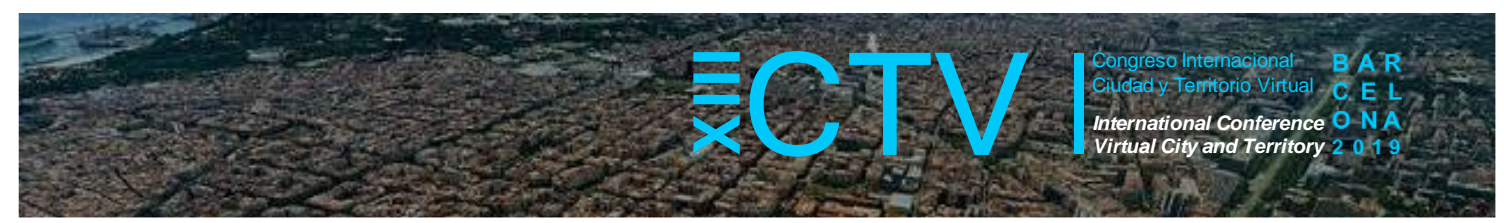

Paulo. The key research question seeks to answer if in the DECB in Bogotá and in the OUCAE in São Paulo advances were made in the materialization of social and spatial justice, based on the progress that has been made in decreasing five specific problems related to particular features of urbanization and development in Latin America: 1. The reduction of private appropriation of land value resulting from public action; 2 . The consolidation of concepts as onerous granting of the right to build, and equitable distribution of burdens and benefits; 3 . The shift of the uneven and often inequitable patterns of distribution of urban infrastructure; 4 . The minimization of negative effects of socio-spatial segregation; and 5. The decrease of informality in access to urban land. For the purpose presented above, it was assumed at first that progress was made in the materialization of socio-spatial justice forms in Bogotá and in São Paulo, mainly, because during its implementation, Land value capture was considered as a process that involves actions before and after recovery and in which the distributive principle and the redistributive goal prevails in both case studies. The methodology used a comparative analysis within three levels of analysis: First, at the general level, were examined concepts, laws and instruments related to the subject of research in Colombia and Brazil; second, at the specific level, the impact of land value capture instruments studied in the materialization of socio-spatial justice in Bogotá and São Paulo was analyzed in three moments: a) the action prior to recovery, b) the action of recovery, and c) the post-recovery action. Finally, at the synthesis level, a comparison of the results in the materialization of socio-spatial justice is made. As a result, it was possible to observe that, the hypothesis proposed was partially confirmed; as, in the first action, prior the recovery of land value capture, it was observed that it is characterized by the unequal distribution of urban infrastructure and the differential value of land, which leads to the need to define mechanisms that seek the reduction of inequalities in the distribution of income and wealth in society. In the second action, the recovery of the land value capture, progress was made in the fight against private appropriation of land value and in the consolidation of the distributive principle. Finally, it was found that a practical contribution resulting from the post-recovery action of land value capture, it is related to the idea that the resources obtained must be primarily allocated to serve the population with lower incomes, either in the same areas where the projects were carried out, as well, in the other areas of the municipality that are not benefited by general budgets of Latin American cities.

Palabras Clave: instrumentos de recuperación de plusvalías urbanas; justicia socioespacial; Bogotá; Sao Paulo

Key words: land value capture instruments; socio-spatial justice; Bogotá; São Paulo

\section{Introducción}

Alterman (2012, p. 755, traducción propia) señala que "pocas cuestiones en la planificación urbana son tan universales como la relación bipolar entre la regulación y los valores de la tierra" y que "este tema tiene profundas implicaciones económicas, sociales y de justicia distributiva". Precisamente, en América Latina, algunos autores han investigado la relación entre la recuperación de plusvalías y las desigualdades urbanas (Furtado, 1999, 2010, 2014; Jaramillo, 2008, 2009).

Por ejemplo, Furtado subraya el hecho que la recuperación de plusvalías "permite redistribuir ventajas especiales asignadas privadamente" y que eso "es algo que no puede ser evaluado independientemente de un conjunto de cuestiones que involucra, entre otras cosas, donde esas ventajas especiales se asignan" (Furtado, 2014, pág. 417, traducción propia). Este planteamiento está estrechamente relacionado con la posibilidad de aumentar la oferta de terrenos dotados de la infraestructura urbana necesaria, que puede financiarse utilizando recursos derivados de la recuperación de plusvalías urbanas resultantes de la acción del Estado (Smolka; Biderman, 2012).

En las ciudades de América Latina, esta idea coincide con dos aproximaciones de Jaramillo $(2008,2009)$ en las que estudia la manera en que se distribuye la infraestructura en el territorio de las ciudades latinoamericanas. El primero de estos enfoques está asociado al concepto de socialización de los costos y privatización de los beneficios. El segundo enfoque se refiere al llamado círculo vicioso que resulta del agravamiento de las contradicciones señaladas anteriormente como consecuencia de las características del proceso de crecimiento urbano en América Latina: 


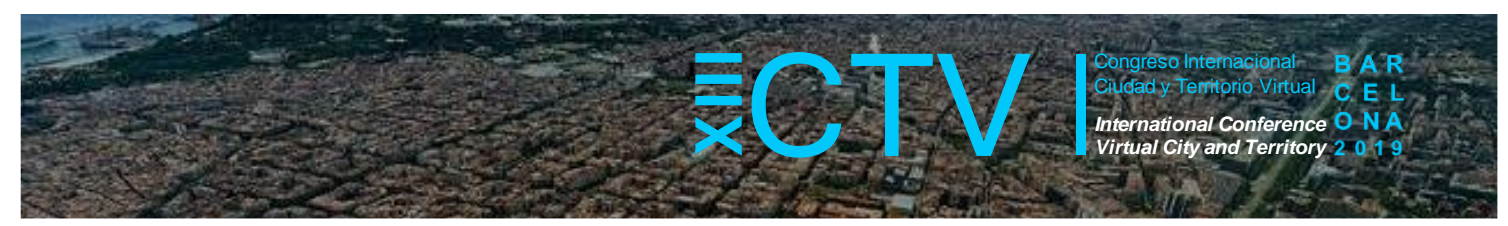

Figura 1. Círculo vicioso del proceso de urbanización en América Latina

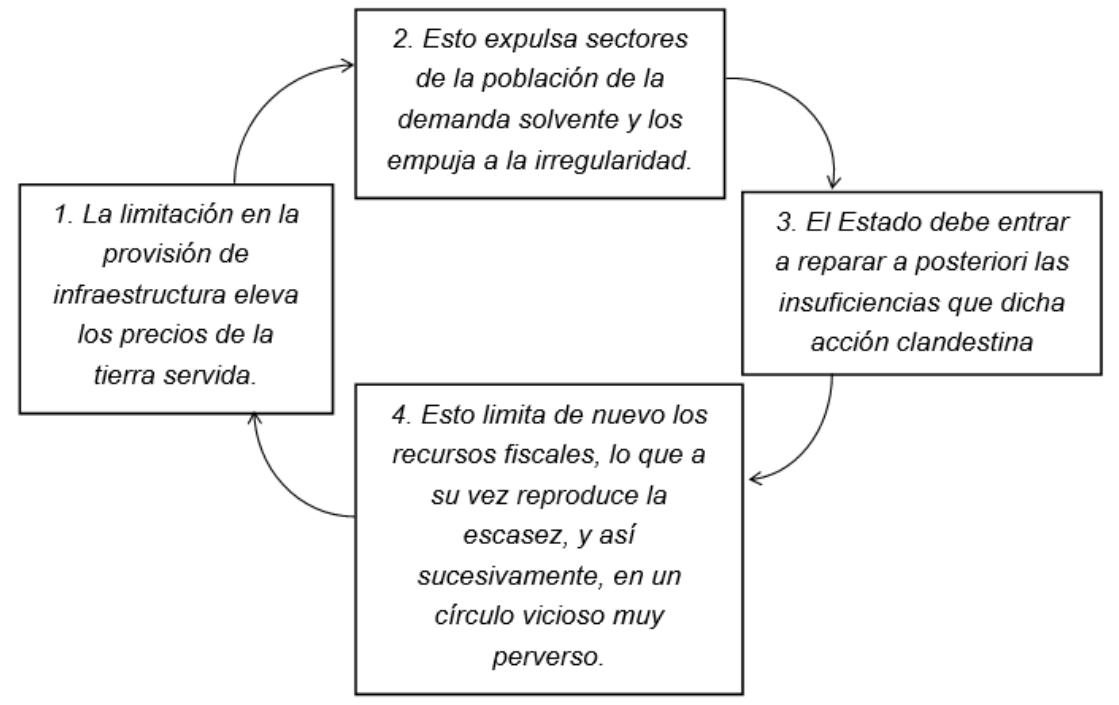

Fuente: Elaboración propia a partir de Jaramillo (2008, p. 50)

Ese autor propone una posible salida a este "injusto" escenario, por medio de la utilización de instrumentos de recuperación de plusvalías urbanas: "[...] si una parte sustancial de los jugosos aumentos en los precios del suelo de los que se apoderan terratenientes originales, especuladores y promotores-especuladores pudiera ser recuperada por el Estado y reorientada a ampliar la oferta de tierra equipada, se podrían tal vez reducir en forma significativa los precios del suelo (aunado a medidas contra la retención de tierras), se permitiría el acceso de los grupos más pobres al equipamiento urbano y se redefiniría la dinámica del promotor de la especulación a la actividad productiva" (Jaramillo, 2009, pág. 438).

De acuerdo a lo anterior, la ponencia, que sintetiza los resultados de mi tesis doctoral " $A$ recuperação de mais-valias fundiárias urbanas e a materialização de justiça socioespacial em Bogotá e São Paulo: Um objetivo, dois casos e um resultado" (Pérez-Moreno, 2018), analiza los impactos de los instrumentos de recuperación de plusvalías en la superación de las desigualdades urbanas en Colombia y Brasil, examinando su relación con la materialización de formas de justicia socioespacial. Para esto, se estudian dos casos relevantes de recuperación de plusvalías urbanas en dichas ciudades: la Distribución Equitativa de Cargas y Beneficios (DECB) definida en el Decreto Distrital 562 de 2014 en Bogotá y la Operación Urbana Consorciada Água Espraiada (OUCAE) aprobada mediante la Ley Municipal 13.260 de 2001 en Sao Paulo.

La materialización de justicia socioespacial fue evaluada a partir de los avances en la disminución de cinco problemáticas relacionadas a los rasgos particulares de los procesos de urbanización en América Latina (Jaramillo, 1992): 1. La disminución de la apropiación privada de la valorización de la tierra resultante de la actuación del Estado; 2. La consolidación de los conceptos de otorgamiento oneroso del derecho de construir y de distribución equitativa de cargas y beneficios; 3 . La alteración de los injustos patrones de distribución de la infraestructura urbana; 4. La reducción de los efectos negativos de la segregación socioespacial (Sabatini, 2003) y 5. La disminución de la informalidad en el acceso al suelo urbano (Smolka, 2010; Veríssimo, 2012; Maldonado, 2007). 


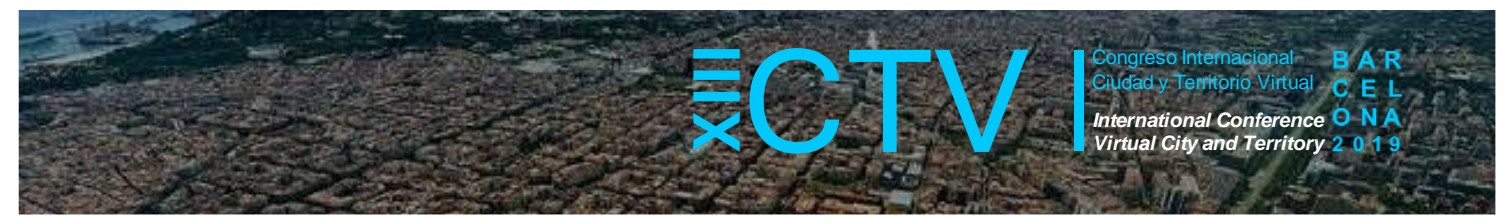

\section{La Distribución Equitativa de Cargas y Beneficios (DECB) definida en el Decreto Distrital 562 de 2014 y la materialización de formas de justicia socioespacial en Bogotá}

Aunque el 26 de agosto de 2013 fue promulgada la Modificación Excepcional del Plan de Ordenamiento Territorial de Bogotá - MEPOT, por medio del Decreto Distrital 364 de 2013, este decreto fue suspendido por el Consejo de Estado de Colombia el 27 de marzo de 2014, obligando a que la norma que continuara vigente fuera nuevamente el Plan de Ordenamiento Territorial (POT) que había sido aprobado en 2003 y compilado en 2004.

Ante este escenario y con el fin de concretar los cambios en el patrón histórico del desarrollo urbano de la ciudad, (expuestas en el Plan de Desarrollo Distrital entre 2012 y 2016), la Alcaldía de Bogotá expidió los decretos distritales 562, 138 y 448, con los cuales se buscaba "renovar las zonas centrales que ofrecen los mejores potenciales urbanos, estimular la vivienda de interés prioritario en estas zonas centrales y la participación de moradores y propietarios en los procesos de renovación urbana" (AMDB; SDP, 2015, pág. 75).

Uno de esos actos administrativos fue el Decreto Distrital 562 del 12 de diciembre de 2014 "Por el cual se reglamentan las condiciones urbanísticas para el tratamiento de renovación urbana, se incorporan áreas a dicho tratamiento, se adoptan las fichas normativas de los sectores con este tratamiento y se dictan otras disposiciones", el cual fue modificado parcialmente por el Decreto Distrital 575 de 2015. El área incorporada al tratamiento urbanístico de renovación urbana en la modalidad de reactivación por el citado decreto fue de 8.991,8 ha., lo que corresponde al $23,4 \%$ del perímetro urbano de la ciudad estipulado en el POT que era de $38.430,19$ ha. (SDP, 2016, BDGC).

El Decreto Distrital 562 de 2014 definió que "la altura de las edificaciones y los índices máximos que se pueden alcanzar en los predios, están limitados por la aplicación de las normas sobre aislamientos, empates, obligaciones urbanísticas, antejardines, retrocesos, provisión del equipamiento comunal privado [...]" (art. 6). Con base en ese tipo de normas se pretendía densificar las áreas más centrales de la ciudad, que tienen las mejores condiciones de infraestructura, equipamiento y densidades consideradas bajas, comparadas con las que se presentan en la periferia sur y occidental de la ciudad donde se localizan las familias de bajos ingresos y en las que la oferta de empleos, infraestructura y equipamientos, es significativamente menor.

A pesar que el Decreto Distrital 562 de 2014 estuvo vigente durante poco más de 14 meses, sus disposiciones normativas produjeron cambios en la recuperación de plusvalías urbanas para la ciudad, especialmente relacionados con la implementación de un sistema de Distribución Equitativa de Cargas y Beneficios (DECB), por medio del cual se lograba concretar la edificabilidad a través del cumplimiento de obligaciones urbanísticas pagadas en los proyectos desarrollados en las áreas de la ciudad incorporadas, por este decreto, al tratamiento urbanístico de renovación en la modalidad de reactivación, sin la necesidad de plan parcial.

\subsection{Acción anterior a la recuperación de plusvalías urbanas}

En el momento anterior a la recuperación de plusvalías urbanas se encontró que, el área considerada en el Decreto Distrital 562 de 2014, hace parte de las zonas de la ciudad en las 


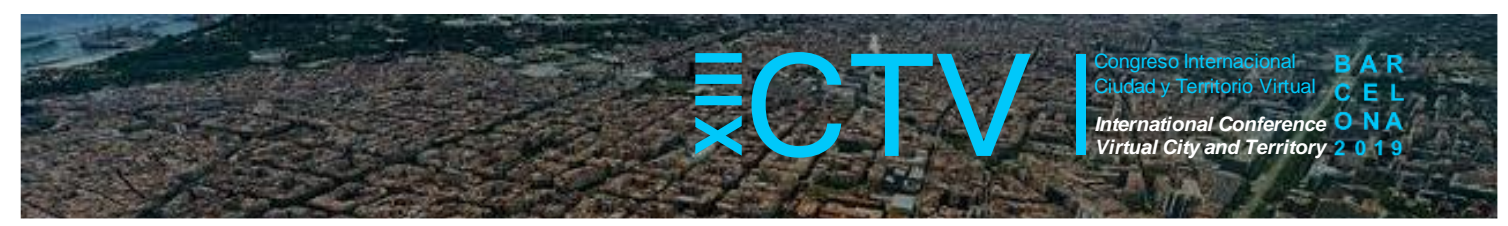

que históricamente fue concentrada la infraestructura urbana de la ciudad, y por consiguiente, los mayores valores del suelo. De esta forma, cualquier mejora en la infraestructura o el aumento de los aprovechamientos de esos terrenos, incrementaría su valor. Esto, por su parte, está estrechamente relacionado a las acciones anteriores del Estado en esas áreas (inversiones públicas y cambios en la normativa urbanística) cuyo consecuente incremento de valor del suelo, no fue históricamente recuperado por el poder público.

\subsection{Acción de recuperación de plusvalías urbanas.}

Con base en el principio del ordenamiento territorial colombiano de distribuir equitativamente las cargas y los beneficios, y como uno de los mecanismos indirectos de recuperación de plusvalías urbanas, el Decreto Distrital 562 de 2014 (art. 16) estableció que, para realizar construcciones con las mayores alturas autorizadas en ese decreto, los proyectos estarían obligados a entregar cargas urbanísticas, para garantizar que no se repitiera el proceso de densificación lote a lote sin cargas urbanísticas (que se presentó en parte de las áreas consolidadas de la ciudad con la aplicación de las normas del del POT de 2004).

Se destaca que esta reglamentación estableció que, para proyectos con Índice de Construcción (I.C.) de hasta 2,0, no serían exigidas obligaciones urbanísticas por edificabilidad, lo que podría ser considerado como un I.C. básico que estaría pagado en los anteriores procesos de urbanización de las áreas consideradas por ese decreto. Esto, constituiría la aplicación de un mecanismo relativamente parecido al coeficiente de aproveitamento básico implementado en Sao Paulo. Sin embargo, en Bogotá, este mecanismo que mezcla conceptos de la Outorga Onerosa do Direito de Construir (OODC) y de las Operações Urbanas Consorciadas (OUC) de Brasil, considerando que el pago por la posibilidad de edificar por encima del I.C. de 2,0 involucra, por un lado, la infraestructura actual que da condiciones básicas para la redensificación propuesta (base de la OODC) y, de otra parte, la construcción de nueva infraestructura adicional, concretizaría las condiciones urbanísticas necesarias para soportar los mayores aprovechamientos (base da OUC).

Contrario a los datos presentados en la recaudación relacionada con el instrumento de participación en plusvalía en Bogotá, el Decreto Distrital 564 de 2014 produjo un aumento significativo de los recursos del Fondo para el pago compensatorio de cesiones públicas para parques y equipamientos, y del Fondo para el pago compensatorio de vías y estacionamientos entre 2014 e 2016. Al analizar los datos de esos fondos durante ese periodo, se encontró que la recaudación pasó de un poco más de USD 13 millones en 2014 a aproximadamente USD 45 millones en 2015 y a un poco más de USD 65 millones en 2016 (SHD, 2016).

Otra de las formas de concretar la distribución equitativa de cargas y beneficios (DECB) estipuladas en el Decreto Distrital 562 de 2014 (art. 22), fue la introducción del pago de obligaciones para servicios públicos. Esto, como otra de las condiciones para poder utilizar las mayores alturas permitidas en este decreto y como otro mecanismo indirecto de recuperación de plusvalías urbanas.

Adicionalmente, a pesar que el Decreto Distrital 562 de 2014 no reglamentó la obligación de destinar terrenos para proyectos de vivienda de interés prioritario (VIP) en proyectos sometidos al tratamiento de renovación urbana, esa exigencia fue reglamentada en el Decreto Distrital 138 de 2015 "Por el cual se reglamenta el artículo 66 del Acuerdo 489 de 2012 para asegurar el cumplimiento del Programa de Vivienda y Hábitat Humanos del Plan de Desarrollo 


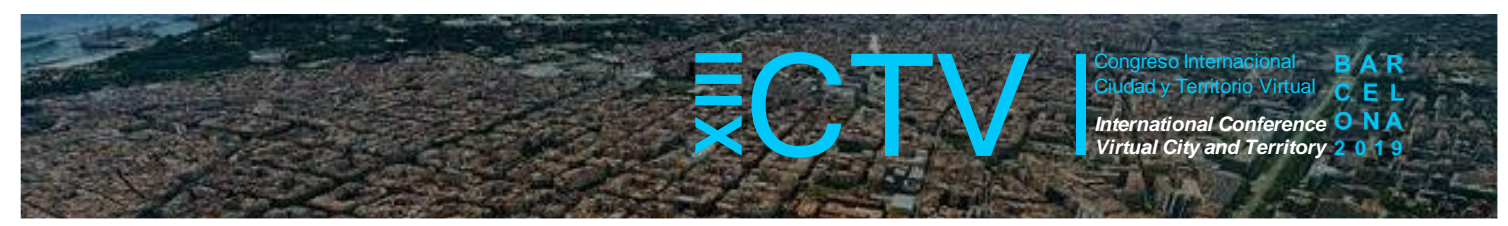

Económico, Social, Ambiental y de Obras Públicas de Bogotá, D. C. 2012-2016, y se adoptan otras disposiciones", el cual tuvo un importante papel en la implementación del Decreto Distrital 562 de 2014.

\subsection{Acción posterior a la recuperación de plusvalías urbanas}

Para analizar la materialización de formas de justicia socioespacial en la acción posterior a la recuperación de plusvalías urbanas realizada a través de la implementación de la DECB del Decreto Distrital 562 de 2014, se utilizaron las siguientes preguntas que orientaron esa parte del análisis:

¿Cuáles recursos fueron redistribuidos para desconcentrar las familias de bajos ingresos y para mejorar su acceso al transporte público?

El Decreto Distrital 575 de 2015 (que modificó el Decreto Distrital 562 de 2014) definió que, en las denominadas áreas especiales, las obligaciones serían cumplidas únicamente por medio de compensación en dinero y que serían destinadas en su totalidad a la adquisición de suelo, construcción y/o financiamiento de la primera línea del Metro de Bogotá.

De esta forma, con el cambio en el Decreto Distrital 562 de 2014, se buscó dirigir recursos provenientes de la recuperación de plusvalías urbanas para mejorar el acceso al transporte público de las familias de bajos ingresos, considerando que la primera línea de metro, que estaba planeada en ese momento, conectaba la zona suroccidental de la ciudad (históricamente ocupada por la población de ingresos bajos y medios) con el centro y el norte de la ciudad (caracterizada por tener la mayoría de puestos de trabajo). Sin embargo, la derogatoria de los Decretos 562 de 2014 y 575 de 2015 por medio del Decreto Distrital 079 de 2016 y los cambios hechos en la destinación de los recursos de los fondos para pagos compensatorios de cesiones públicas para parques, equipamientos, vías y estacionamientos (hechas por el Decreto Distrital 603 de 2016) hicieron que esa destinación específica para la línea de metro de Bogotá fuese modificada hacia una destinación general.

¿Cuáles recursos fueron redistribuidos buscando incluir familias de bajos ingresos en áreas ocupadas por población con mayores ingresos?

Por medio de la utilización combinada de los Decretos 562 de 2014 y 138 de 2015 se consiguieron recursos para proyectos de VIP desarrollados por instituciones públicas, considerando que el Decreto Distrital 138 de 2015 permitía la compensación de la obligación de destinar suelo para este tipo de viviendas por medio de la compra de derechos fiduciarios. De acuerdo con la información suministrada por la Empresa de Renovación y Desarrollo Urbano de Bogotá (ERU, 2017), entre 2015 e 2016 fueron recaudados un poco más de US $\$ 19$ millones resultantes de la compra de los mencionados derechos fiduciarios.

Esta acción conjunta no consiguió redistribuir recursos buscando incluir familias de bajos ingresos en áreas con población de ingresos superiores, considerando que casi $89 \%$ de las inversiones públicas con el dinero recaudado fueron destinadas a proyectos de VIP que se concentraron en las localidades de Bosa e Usme, zonas históricamente ocupadas por familias de bajos ingresos y en las que localizan una cantidad significativa de barrio de origen informal. 


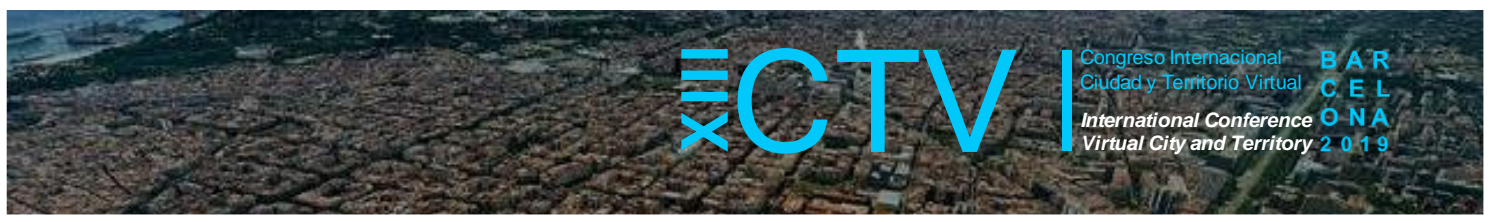

Esa situación podría estar relacionada a la posibilidad de cumplir esta obligación mediante derechos fiduciarios, lo cual, debilita la inversión de estos recursos en las áreas en que son generados, obstruyendo la inserción de VIP en estos mismos lugares.

¿Qué recursos fueron redistribuidos para el mejoramiento y la regularización de barrios de origen informal?

El Decreto Distrital 562 de 2014 no consideró destinar recursos para el mejoramiento y regularización de barrios de origen informal. Parte de los recursos que ingresaron al Fondo Compensatorio de Parques y Equipamientos fueron destinados para mejorar e incrementar la infraestructura de parques y equipamientos de la ciudad, sin embargo, no fue posible obtener información respecto a la localización de estas inversiones.

¿El valor de la tierra disminuyó por la recuperación de plusvalías urbanas?

De conformidad con la información de los Rangos de Valores de Referencia del Suelo para Bogotá en 2014 y 2016 (antes y después de la recuperación de plusvalías urbanas realizada por medio del Decreto Distrital 562 de 2014), se puede afirmar que, en buena parte de las áreas reglamentadas en este decreto, el valor del suelo aumentó (UAECD, 2016). Sin embargo, ese incremento pudo estar relacionado también con otros importantes factores como la escasa oferta de tierras urbanizadas en la periferia de la ciudad y las dificultades en el desplazamiento entre las áreas centrales y la periferia, las cuales se agudizaron en este periodo.

Sin embargo, en las entrevistas con los actores involucrados en la implementación del Decreto Distrital 562 de 2014 se resaltó que, en algunas transacciones de compraventa, los dueños de la tierra solicitaron valores menores por su suelo, después de la expedición de ese decreto, considerando las mayores obligaciones urbanísticas (Pérez-Moreno, 2018). Sin embargo, no fue posible levantar evidencias concretas de esa situación.

\section{¿Qué recursos fueron redistribuidos para generar suelo urbanizado de calidad?}

El Decreto Distrital 562 no consideró destinar recursos para generar suelo urbanizado de calidad, sin embargo, se puede afirmar que su intención fue la de redensificar áreas centrales de la ciudad, las cuales, a pesar de estar urbanizadas tenían densidades de ocupación consideradas bajas, mientras que áreas periféricas de la ciudad presentaban deficiencias en la infraestructura urbana y altas densidades de ocupación.

\section{La concreción de formas de justicia socioespacial en la Operación Urbana Consorciada Água Espraiada (OUCAE) aprobada mediante la Ley Municipal 13.260 del 2001 en Sao Paulo}

La Operación Urbana Consorciada Água Espraiada (OUCAE) ocupa un área aproximada de 1400 ha. en la zona oeste y sur de Sao Paulo (Brasil). En su eje norte-sur se localizan las Avenidas Marginal Pinheiros y las Avenidas Engenheiro Luís Carlos Berrini e Doutor Chucri Zaidan, y, en su eje oriente-occidente se encuentra la cuenca hidrográfica de la corriente Água Espraiada, en la que se localizan actualmente cerca de cuarenta favelas y parte de la Avenida Jornalista Roberto Marinho. 


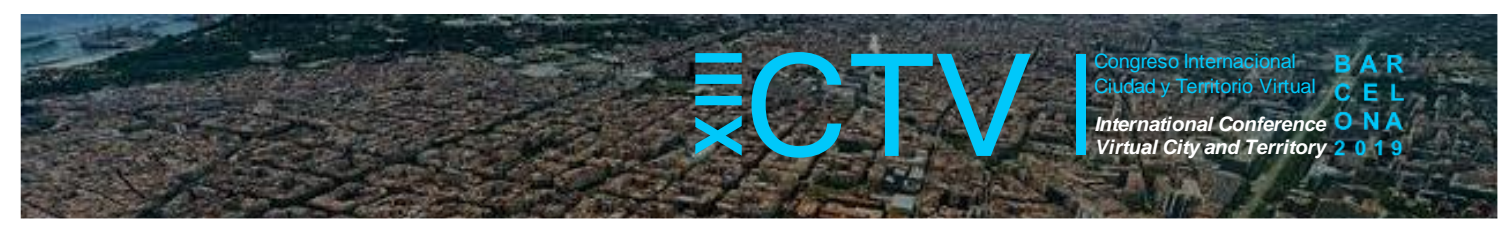

La OUCAE en Sao Paulo fue aprobada por la Prefectura de Sao Paulo por medio de la Ley Municipal 13.260 de 2001, que fue posteriormente modificada por la Ley Municipal 15.416 de 2011. La principal innovación introducida por la OUCAE en 2001 fue la definición de ZEIS (Zonas Especiais de Interesse Social) dentro del perímetro de la operación, además, definió la intención de construir vivienda de interés social (HIS por su sigla en portugués - Habitação de Interesse Social) para atender a las familias afectadas por las obras e intervenciones de esa operación urbana. Posteriormente, en la ley que alteró la OUCAE en 2011, se incluyó la obligación de invertir, como mínimo, 10\% de los recursos obtenidos en la operación urbana, para la construcción de HIS, también para las familias que resultasen afectadas por la OUCAE.

La OUCAE constituyó un grupo de gestión encargado de formular y acompañar los planes y proyectos urbanísticos definidos en el programa de intervenciones, de sugerir la revisión de la ley de esa operación urbana consorciada y de su control general (art. 20). Esto, para cumplir con uno de los contenidos mínimos establecidos por el Estatuto de la Ciudad para las leyes que aprueban OUC: el control compartido con la sociedad civil.

\subsection{Acción anterior a la recuperación de plusvalías urbanas}

En el momento anterior a la recuperación de plusvalías urbanas se encontró que, así como en la mayoría de las ciudades brasileras, en Sao Paulo, donde se desarrolló la OUCAE la distribución diferenciada de la infraestructura por el poder público (que fue construida específicamente en las áreas en las que se localiza la población de mayores ingresos) está relacionada estrechamente con los aumentos en el valor del suelo. En este escenario, las normas diferenciadas, los escasos impuestos territoriales sobre las ganancias inmerecidas resultantes de esos incrementos en el valor de la tierra y la injusta distribución de los servicios urbanos dan características particulares a las acciones públicas anteriores a la recuperación de plusvalías urbanas en la OUCAE.

\section{2 $\quad$ Acción de recuperación de plusvalías urbanas}

El éxito fiscal de la OUCAE ha sido relevante. De acuerdo con el Resumen Financiero de esta operación (SP-URBANISMO, 2017), los recursos recaudados desde 2001 hasta octubre de 2017 fueron aprox. USD 1.190 millones. De ese dinero, USD 1.076 millones fueron invertidos en la operación urbana $(90,5 \%$ del total) y USD 113 millones todavía permanecían sin ser invertidos (lo que equivale a $9,8 \%$ de total). Esos recursos colectados por la OUCAE provinieron de la venta de CEPAC (Certificados de Potencial Adicional de Construcción) por medio de subastas $(73,4 \%)$ y de los rendimientos financieros $(25,2 \%)$, esto por haber invertido, en el sistema financiero, parte de los ingresos resultantes de la venta de los CEPAC antes de invertirlos en la OUCAE.

El dinero recaudado en la OUCAE corresponde a un poco más del $7 \%$ de los ingresos que estimó la Ley de Presupuesto Fiscal del Municipio de Sao Paulo para 2017, que fueron cercano a los USD 16.700 millones (Sao Paulo-Municipio, 2016), y equivale a 1,6 veces los recursos recaudados como contrapartida por la venta de derechos de construir hasta septiembre de 2019, a través de otros de los instrumentos de recuperación de plusvalía que tiene la ciudad de Sao Paulo: el OODC (Otorgamiento Oneroso del Derecho de Construir), que recaudó USD 730.767 según el portal GeoSampa (Prefectura de Sao Paulo, 2017). Además, es preciso destacar que la OUCAE fue la operación urbana consorciada que más dinero recaudó en la 


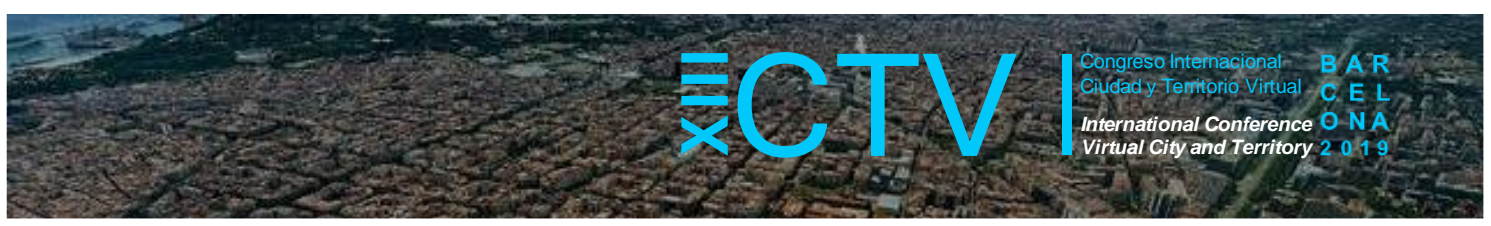

ciudad de São Paulo, con $53,7 \%$ del total de ingresos de las cuatro intervenciones de ese tipo que están desarrollándose en la ciudad (total que corresponde hasta 2017 a valor cercano de USD 2.215 millones.

\subsection{Acción posterior a la recuperación de plusvalías urbanas.}

Para examinar la acción posterior a la recuperación de plusvalías urbanas en la OUCAE y su papel para concretar formas de justicia socioespacial, se utilizaron las mismas preguntas que en el anterior caso de Bogotá:

¿Cuáles recursos fueron redistribuidos para desconcentrar las familias de bajos ingresos y para mejorar su acceso al transporte público?

Respecto a la distribución de recursos destinados a desconcentrar a las familias de bajos ingresos, no fue posible identificar inversiones directas de la OUCAE para ese grupo de población, además del dinero destinado a la construcción de HIS para la población afectada por la operación urbana.

En relación a la redistribución de recursos para el mejoramiento del acceso al transporte público de las familias de bajos ingresos, se destaca la inversión de USD 120 millones en obras de transporte colectivo (Metro) que indirectamente benefician a este grupo de población no solo dentro de la operación urbana. Esta inversión corresponde al $10 \%$ a los gastos del flujo financiero hasta octubre de 2017 (SP-URBANISMO, 2017).

¿Cuáles recursos fueron redistribuidos buscando incluir familias de bajos ingresos en áreas con la población ingresos mayores?

Al analizar la distribución de recursos recaudados en la OUCAE, sobresale la cantidad de recursos invertidos para HIS, que, junto con el dinero que aún no se ha gastado (que tiene una destinación específica para este tipo de vivienda) y los recursos que fueron destinados para el pago de expropiaciones para HIS suman en total USD 280 millones que corresponden al 23,6\% de la totalidad de los recursos recaudados (SP-URBANISMO, 2017).

Además de eso, se destaca la relevante cantidad de unidades de HIS que fueron y serán construidas en la OUCAE para atender a la población de las favelas afectadas por las intervenciones previstas en la operación. De acuerdo con los datos presentados en el Grupo de Gestión de la OUCAE del 29 de noviembre de 2017 (SP-URBANISMO, 2017b), hasta ese momento 710 unidades de HIS habían sido entregadas, 4.294 estaban en construcción y 4.293 más estaban previstas, para un total de 9.297 unidades de HIS, siendo la operación urbana consorciada con la mayor producción de viviendas de ese tipo en Sao Paulo.

Se destacan los conjuntos de HIS Jardim Edite I y II (con 252 y 68 unidades habitacionales) que fueron construidos en el Sector Berrini, distrito que se caracteriza por tener población de altos ingresos. La construcción de esos dos conjuntos permitió que una parte de la población que vivía en lo que restó de la favela Jardim Edite pudiese permanecer en la misma zona, sin embargo, esto involucró un proceso largo de negociación y resistencia de sus habitantes, que finalmente resultó en un hecho capaz de establecer un nuevo paradigma para situaciones similares en esta y en otras OUC. 


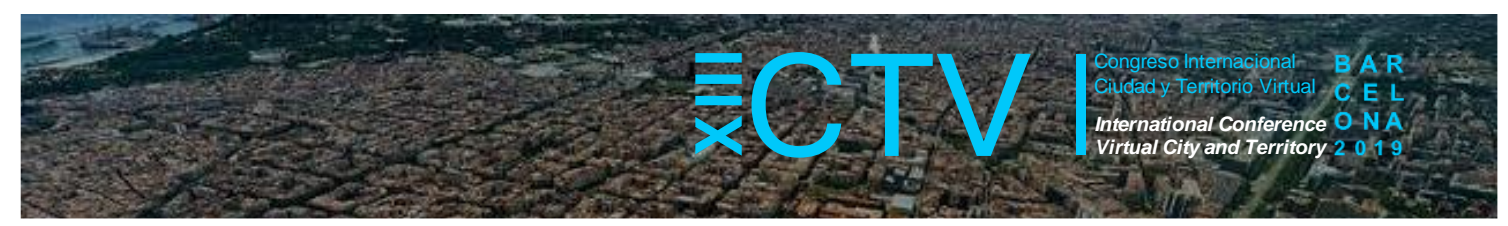

Así queda claro que, incluso sin conseguir cambios significativos en la inserción de familias de bajos ingresos en áreas con población de mayores ingresos, la OUCAE finalmente incorporó la idea que la población de las favelas pudiese vivir en conjuntos de HIS mucho más próximos a las áreas originales de esos asentamientos.

Los hechos anteriores evidencian que, a pesar de los avances en la reglamentación y en la gestión, la materialización de justicia socioespacial por medio de la construcción de HIS en la OUCAE es apenas el inicio de una iniciativa que ahora parece tener un camino más claro. Esto, considerando que en los próximos años se espera la finalización de una cantidad significativa de unidades de HIS en la OUCAE y que la obligación de construir este tipo de vivienda ya fue incluida en otras OUC, como en Água Branca, en la cual se espera construir la HIS integrada a proyectos habitacionales destinados a población de mayores ingresos y cercana a equipamientos y espacios públicos.

¿Cuáles recursos fueron redistribuidos para el mejoramiento y la regularización de barrios de origen informal?

En relación a la redistribución de recursos para el mejoramiento y la regularización de barrios de origen informal, es necesario aclarar que la Ley Municipal 13.260/2001 de la OUCAE, definió la realización de mejoras y de obras de urbanismo que garantizaran la atención a los habitantes de estos barrios, el reasentamiento de las familias que fueron afectadas por las obras previstas en la operación urbana, y el desarrollo de unidades de HIS, como parte del programa de la operación y en cumplimiento a los objetivos de esta intervención. Sin embargo, los recursos destinados a realizar estos objetivos de la OUCAE se destinaron únicamente a la construcción de las unidades de HIS para las familias que habitaban en esas áreas y que resultaron impactadas por la operación. La estrategia principal de la OUCAE con respecto a los barrios informales (favelas) localizados en su perímetro fue el traslado de familias y su reasentamiento en las nuevas viviendas de interés social, en lugar de realizar las obras de mejoramiento o de regularización urbana.

¿El valor de la tierra disminuyó por la recuperación de plusvalías urbanas?

Al examinar los cambios en el valor catastral promedio del suelo entre 2001 y 2016 , se observa que los valores de estos terrenos tuvieron un aumento considerable tanto en la ciudad de Sao Paulo (MSP) como en la OUCAE, representando en el caso de MSP, un aumento del $724.2 \%$ y en la OUCAE del $686.6 \%$ (Pérez-Moreno, 2018). Este menor porcentaje en la OUCAE podría estar relacionado con la implementación de un sistema de recuperación de plusvalías en la operación, en la cual, la introducción de un mecanismo como los CEPAC asociados a la implementación de un coeficiente de aprovechamiento básico, tendió a reducir el valor del suelo. Sin embargo, otros factores como los retrasos en la finalización de las obras de esta operación urbana y la valorización significativa de algunas áreas de la ciudad debido al aumento de la actividad del mercado inmobiliario en este período, también deben ser consideradas.

A pesar del aumento significativo en el valor catastral promedio del suelo entre 2001 y 2016 y de las intervenciones urbanas realizadas en OUCAE, las diferencias en los precios del suelo dentro de esta operación no solo se mantuvieron, sino que, se agudizó la concentración de 


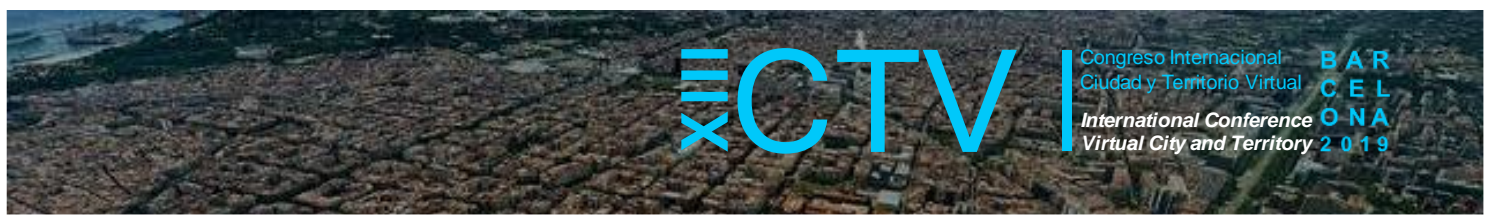

lotes de alto valor en los sectores Berrini, Chucri Zaidan y Brooklin, sectores en los se terminaron la mayoría de las obras públicas de la OUCAE.

¿Qué recursos fueron redistribuidos para generar suelo urbanizado de calidad?

Incluso sin destinar recursos para generar nueva tierra urbanizada de calidad, la OUCAE tuvo la intención de mejorar urbanísticamente un área en que fue autorizada la redensificación. Sin embargo, hasta ahora, los cambios más significativos están relacionadas con la construcción de edificios privados, mientras que las intervenciones urbanísticas quedaron concentradas en obras viales. Un ejemplo de esa priorización vial fue la construcción del viaducto denominado Ponte Estaiada que fue entregado en 2008 y en el que fueron invertidos USD 76 millones resultantes de la venta de CEPAC, lo que equivale a $6,4 \%$ de la totalidad de recursos recaudados en la OUCAE.

\section{Conclusiones}

La hipótesis propuesta en la investigación fue confirmada parcialmente. Esto, considerando que, en los casos estudiados, la acción anterior a la recuperación de plusvalías urbanas estuvo caracterizada por la distribución desigual de la infraestructura urbana y por la valorización diferenciada de la tierra.

En el caso de la acción de recuperación de plusvalías urbanas, se presentaron avances en el combate a la apropiación privada de la valorización de la tierra y en la consolidación del principio distributivo.

Fue encontrado, además, que un aporte práctico resultado del análisis de la acción posterior a la recuperación de plusvalías urbanas, está relacionado a que con la inversión de los recursos obtenidos sean destinados prioritariamente en la atención de la población de menores ingresos, ya sea en las mismas áreas donde fueron realizados los proyectos, como en las otras áreas de la ciudad que no son beneficiadas por los presupuestos generales de los municipios.

Agradecimientos: Esta ponencia es fruto de la tesis de doctorado titulada "A recuperação de mais-valias fundiárias urbanas e a materialização de justiça socioespacial em Bogotá e São Paulo: Um objetivo, dois casos e um resultado", realizada para obtener el título de Doctor en Arquitectura y Urbanismo de la Universidad Federal Fluminense (Niterói, RJ, Brasil), la cual elaboré como parte de los compromisos de la beca PEC-PG de la Coordinación de Perfeccionamiento del Personal de Nivel Superior (CAPES) del Gobierno de Brasil. Agradezco, especialmente a la Arq. Dra. Fernanda Furtado de Oliveira e Silva, quien fue la directora de la tesis mencionada.

Conflicto de Intereses: El autor declara que no hay conflicto de intereses.

\section{Bibliografía}

AMBD - Alcaldía Mayor de Bogotá; SDP - Secretaría Distrital de Planeación (2015). Planeación en Bogotá Humana. Colección Bogotá Humana. Recuperado de: http://www.sdp.gov.co/portal/page/portal/PortalSDP/Noticias2014/SDP realiza lanzamiento de su coleccion de libros Bogota Hum/Planeacion en la Bogota Humana.pdf 


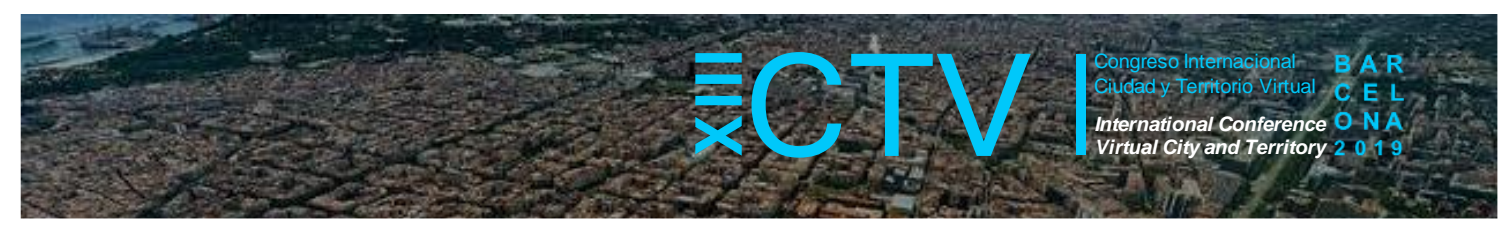

Alterman, R. (2012). Land-use Regulations and Property Values: The "Windfalls Capture" Idea Revisited. En: N. Brooks; K. Donaghy; G. Knaap, (Eds.). The Oxford Handbook of Urban Economics and Planning. (pp. 755-786). New York: Oxford University Press.

Decreto Distrital 562 de 2014 Por el cual se reglamentan las condiciones urbanísticas para el tratamiento de renovación urbana, se incorporan áreas a dicho tratamiento, se adoptan las fichas normativas de los sectores con este tratamiento y se dictan otras disposiciones. Recuperado de: http://www.alcaldiabogota.gov.co/sisjur/normas/Norma1.jsp?i=60137

Decreto Distrital 575 de 2015. Por el cual se modifica el Decreto Distrital 562 de 2014 y se dictan otras disposiciones. Recuperado de: http://www.alcaldiabogota.gov.co/sisjur/normas/Norma1.jsp?i=64187\#1

ERU (2017). Respuesta Petición № 2176162017. Por: I. Florez, Subgerente de Gestión Inmobiliaria da Empresa de Renovación y Desarrollo Urbano de Bogotá-ERU.

Furtado, F. (1999). Recuperação de mais-valias fundiárias urbanas na América Latina: debilidade na implementação, ambigüidades na interpretação (Tesis Doctoral en Arquitectura y Urbanismo). Universidad de Sao Paulo, Sao Paulo.

Furtado, F. (2010). Reformulación de las políticas de recuperación de plusvalías en América Latina. En: M, Smolka, y L. Mullahy. Perspectivas urbanas - Temas críticos de políticas de suelo en América Latina. (p. 229-235). Cambridge: Lincoln Institute of Land Policy.

Furtado, F. (2014). Uma revisão das bases conceituais para um sistema de instrumentos de política fundiária urbana. En: M. Costa, y S. Maria Laís Pereira (Org.) Produção e gestão do espaço - 10 anos de PPGAU/UFF. (pp. 409-426). Niterói: FAPERJ; Casa 8.

Jaramillo, S. (1992). El desenvolvimiento de la discusión sobre la urbanización latinoamericana: hacia un nuevo paradigma. En: S. Jaramillo, y LM. Cuervo (Eds), Urbanización Latinoamericana (pp. 9-44). Bogotá: Escala.

Jaramillo, S. (2008). Reflexiones sobre la "informalidad" fundiaria como peculiaridad de los mercados del suelo en las ciudades de América Latina. Revista Territorios, (vol. 18), 11-53.

Jaramillo, S. (2009). Hacia una teoría de la renta del suelo urbano. Bogotá: Ediciones Uniandes.

Lei 13.260 de 28 de dezembro de 2001. Estabelece diretrizes urbanísticas para a área de influência da atual Avenida Água Espraiada, de interligação entre a Avenida Nações Unidas (Marginal do Rio Pinheiros) e a Rodovia dos Imigrantes, cria incentivos por meio de instrumentos de política urbana para sua implantação, institui o Grupo de Gestão, e dá outras providências. Recuperada de:

http://www.prefeitura.sp.gov.br/cidade/secretarias/desenvolvimento urbano/sp urbanismo/oper acoes urbanas/agua espraiada/index.php? $\mathrm{p}=19602$

Maldonado M. (2007). ¿Es posible anticipar-se a la urbanización informal? Reflexiones a partir de la Operación Urbanística Nuevo Usme, y del macroproyecto Ciudadela Gonzalo Vallejo Restrepo Pereira (Colombia). En: A. Larangeira (Ed.), Regularización de Asentamientos Informales en América Latina (pp. 324-343). Cambridge: Lincoln Institute of Land Policy.

Pérez-Moreno, O. (2018). A recuperação de mais-valias fundiárias urbanas e a materialização de justiça socioespacial em Bogotá e São Paulo: Um objetivo, dois casos e um resultado (Tesis Doctoral em Arquitetura e Urbanismo). Universidade Federal Fluminense, Niterói, Rio de Janeiro. 


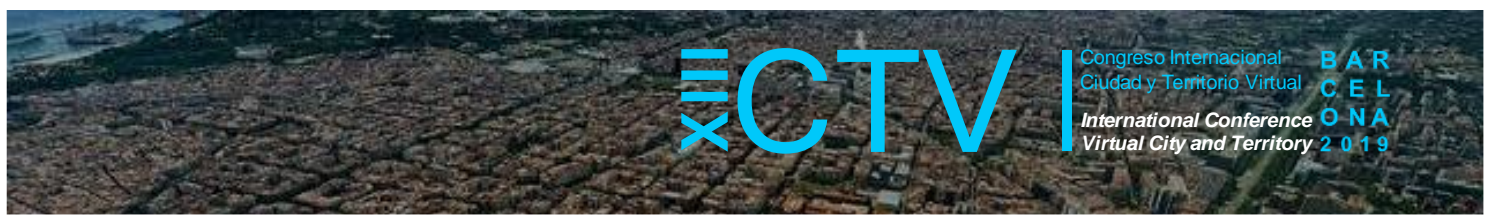

Prefectura de São Paulo (2017). Sitio de internet Dados abertos do Portal de consulta del Mapa Digital da Cidade de São Paulo - GEOSAMPA. São Paulo: 2017a. Recuperado de: http://geosampa.prefeitura.sp.gov.br/PaginasPublicas/ SBC.aspx

Sabatini, F. (2003). La segregación social del espacio en las ciudades de América Latina. Washington D.C: Banco Interamericano de Desarrollo - BID.

SDP-Secretaría Distrital de Planeación. (2016). Base de Datos Geográfica Corporativa-BDGC. Recuperado de: http://www.alcaldiabogota.gov.co/sisjur/normas/Norma1.jsp?i=64187\#1

SHD -Secretaría de Hacienda Distrital-SHD (2016). Informes de Ejecución Presupuestal. 2016. Recuperado de: http://www.shd.gov.co/shd/informes-presupuestales.

Smolka, M. (2010). Informalidad, pobreza urbana y precios de la tierra. En: M. Smolka, y L. Mullahy. Perspectivas urbanas-Temas críticos de políticas de suelo en América Latina (pp. 7178). Cambridge: Lincoln Institute of Land Policy.

Smolka, M., Biderman, C. (2012). Housing Informality: An Economist's perspective on urban planning. n: N. Brooks, K. Donaghy, y G-J. Knaap (Eds.), The Oxford Handbook of Urban Economics and Planning (pp. 814-833). New York: Oxford University Press.

SP-Urbanismo (2017). Operação Urbana Consorciada Água Espraiada - Resumo Financeiro da Movimentação até 31/10/2017. Recuperado de: http://www.prefeitura.sp.gov.br/cidade/secretarias/upload/desenvolvimento urbano/sp urbanis mo/AGUA ESPRAIADA/2017/OUAguaEspraiadaFinanceiroOut17 Publicacao.pdf

SP-Urbanismo (2017b). Operação Urbana Consorciada Água Espraiada - 42a Reunião Ordinária do Grupo de Gestão - 29/11/2017. Recuperado de: http://www.prefeitura.sp.gov.br/cidade/secretarias/upload/desenvolvimento urbano/sp urbanis mo/AGUA ESPRAIADA/2017/GGOUCAE 46a RO 20171206 apresentacao.pdf

Veríssimo, A. (2012). Programa de regularización y formación de las plusvalías en las urbanizaciones informales. En: T. Bolivar, J. Espinosa-Erazo (Coord.). Dimensiones del hábitat popular latinoamericano (pp. 45 - 65). Quito: FLACSO.

UAECD - Unidad Administrativa Especial de Catastro Distrital de Bogotá (2016). Así evoluciona Bogotá. Análisis inmobiliario 2008-2016. Recuperado de: http://idecabogota.maps.arcgis.com/apps/MapJournal/index.html?appid=656ad05d35d0499983 dcede0278061a6\# 